\title{
Colector parabólico compuesto para therma solar
}

\author{
Recepción: Junio de $2006 /$ Aceptación: Octubre de 2006
}

(1) Werner Pacheco Luján

\section{RESUMEN}

El Colector Parabólico Compuesto (CPC) está constituido de 2 superficies parabólicas cuyo

foco es el mismo, en ese foco se coloca un tubo de cobre que se pinta de negro para transformar la luz solar que llega a la superfície de cobre en calor; el CPC concentra la luz en el foco y puede aún recibir rayos con incidencia oblicua de acuerdo a su ángulo de aceptación el que puede ser aumentado con aletas de cobre soldadas a los tubos de cobre. Este conjunto permite tambien recibir y concentrar luz difusa de un día nublado. 4 Colectores de 1,00 m x 1,25 $\mathrm{m}$ son conectados a una therma eléctrica de 500 litros la que almacenará el agua calentada por los colectores, por efecto termosifón ésta tiene también resistencia eléctrica por si hay varios días sin sol.

Palabras Clave: Therma Solar, colectores, parabólicas compuestas.

Composite Parabolic Colector for SOLAR THERMA

ABSTRACT

The Composed Parabolic Collector (CPC) is made up of two parabolic surfaces whose center is the same. In such a center, a black-painted copper tube is placed to transform the sunlight that comes to the copper surface heating it; the CPC concentrates the light in the center, and it can even receive rays with oblique incidence according to its angle of acceptance, the one that could be increased with copper fins welded to the copper tubes. This set also allows to receive and to concentrate diffuse light in a cloudy day. Four Collectors of $1.00 \mathrm{~m} \times 1.25 \mathrm{~m}$ are connected to an electrical therma of 500 liters, that will store the water warmed up by the collectors, and through the themosiphon effect, this one has also has electrical resistance in case there were several non-sunny days.

Key words: Solar therma, collectors, parabolics, composed.
I N T ROD U C C I Ó N

Si proyectamos y construímos un sistema que funciona perfectamente pero es caro y muy poca gente puede adquirirlo no hemos hecho practicamente nada, mas aun la crisis económica que actualmente se vive, nos obliga a extremar esfuerzos para desarrollar uma therma solar barata, en ese propósito lo primero que tenemos que elegir es el método que vamos a utilizar de acuerdo a la realidad de nuestro país. Perú exporta cobre pero no lo procesa y los tubos de cobre y las aletas que se necesitan son importados y por ende caros, cubrir toda la superfície receptora con tubos y aletas de cobre como se hace en el colector plano va a resultar caro, por lo tanto la estrategia adecuada es reducir la superficie de cobre a expensas de superficie reflectora de aluminio pulido que es más barato.

La superfície de cobre en el CPC es un tercio del convencional, eso va permitir que se disminuya tambien la cantidad de pintura negra a utilizar. Por otra parte la pintura negra es buen adsorbente del calor cuando hay sol, pero tambien es buen emisor del calor cuando no hay sol y son mas las horas sin sol en que el colector va ha estar enfriandose. Con menos cuerpo negro en el colector el sistema se enfría más lentamente y es más eficiente, lo que se comprobó en la therma solar de Yangas en que la temperatura cae sólo unos 5 grados durante la noche.

CONSTRUCCIÓN DE LA THERMA SOLAR

En Europa en que la incidencia del sol es menor y cae con un ángulo de inclinación de más de 30 grados de acuerdo a la inclinación del lugar, la relación área colectora volumen de agua está sobredimensionada y es de 1,00 metro cuadrado de colector para 60 litros de agua. En Lima (12 grados) será de 1,00 metro cuadrado de colector para 100 litros de agua.

Para 500 litros de agua necesitamos un colector de 5 metros cuadrados de área, lo que se consigue con 4 cajas colectoras de $1,25 \mathrm{~m}^{2}$ cada una, como está indicado en el esquema (figura 1).

Las cajas colectoras son de fibra de vidrio: que es buen aislador, son livianas lo que es importante a la hora del transporte y además resistente a las inclemencias del medio exterior a que va a estar expuesto en la azotea. En la industria peruana el procesado de fibra de vidrio está bien desarrollado. En el esquema se muestra una sola caja con 3 tubos de cobre y sus aletas las que están en el foco de cada superficie parabólica (son 3 en cada caja) en las otras 3 cajas el esquema es similar (no se

(1) Doctor en Ingeniería Energética. Profesor de la Facultad de Ingeniería Electrónica y Eléctrica, UNMSM. E-mail:wp139@yahoo.com 


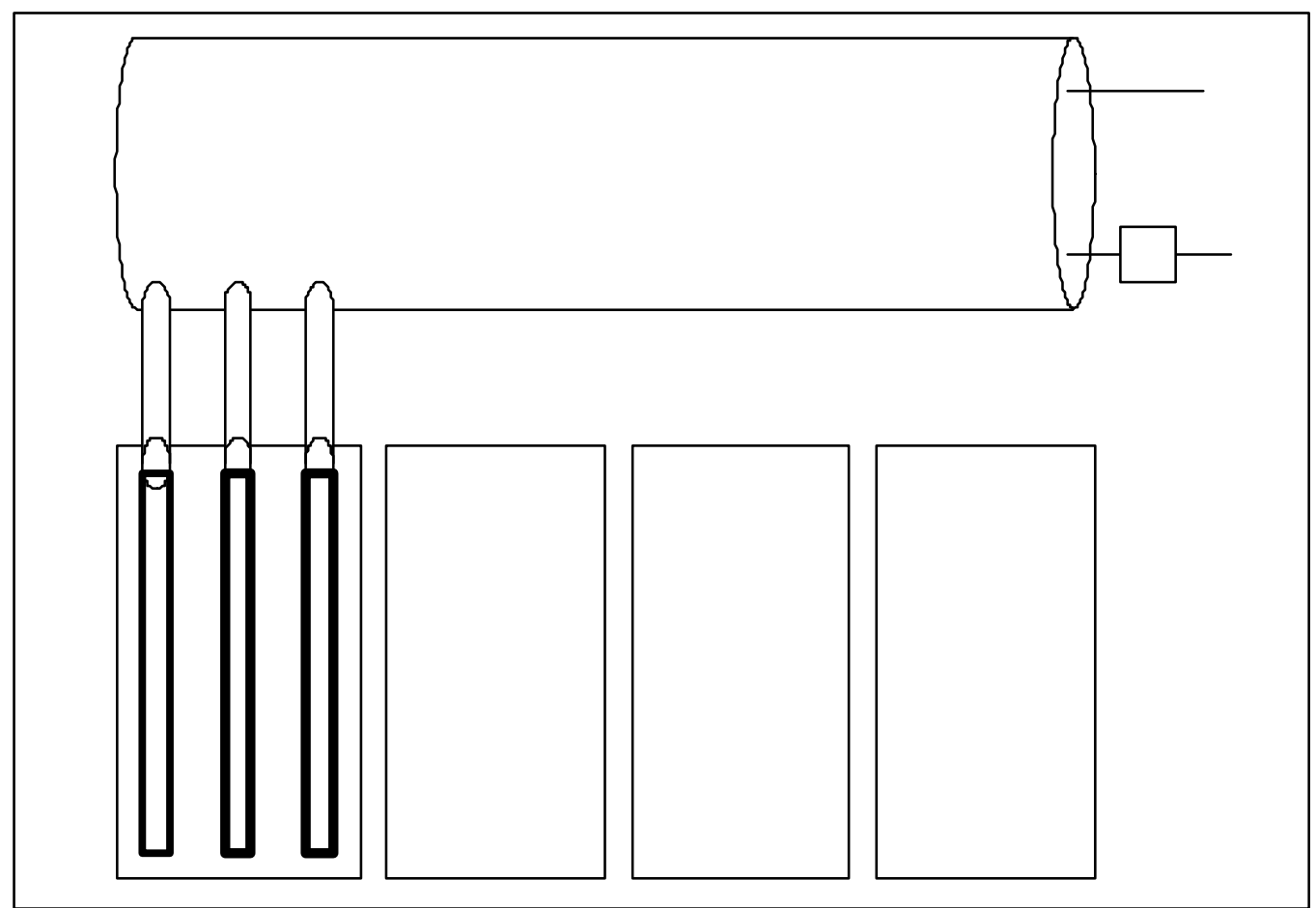

Figura 1. Sistema therma colectores Fuente: Elaboración propia, 2006.

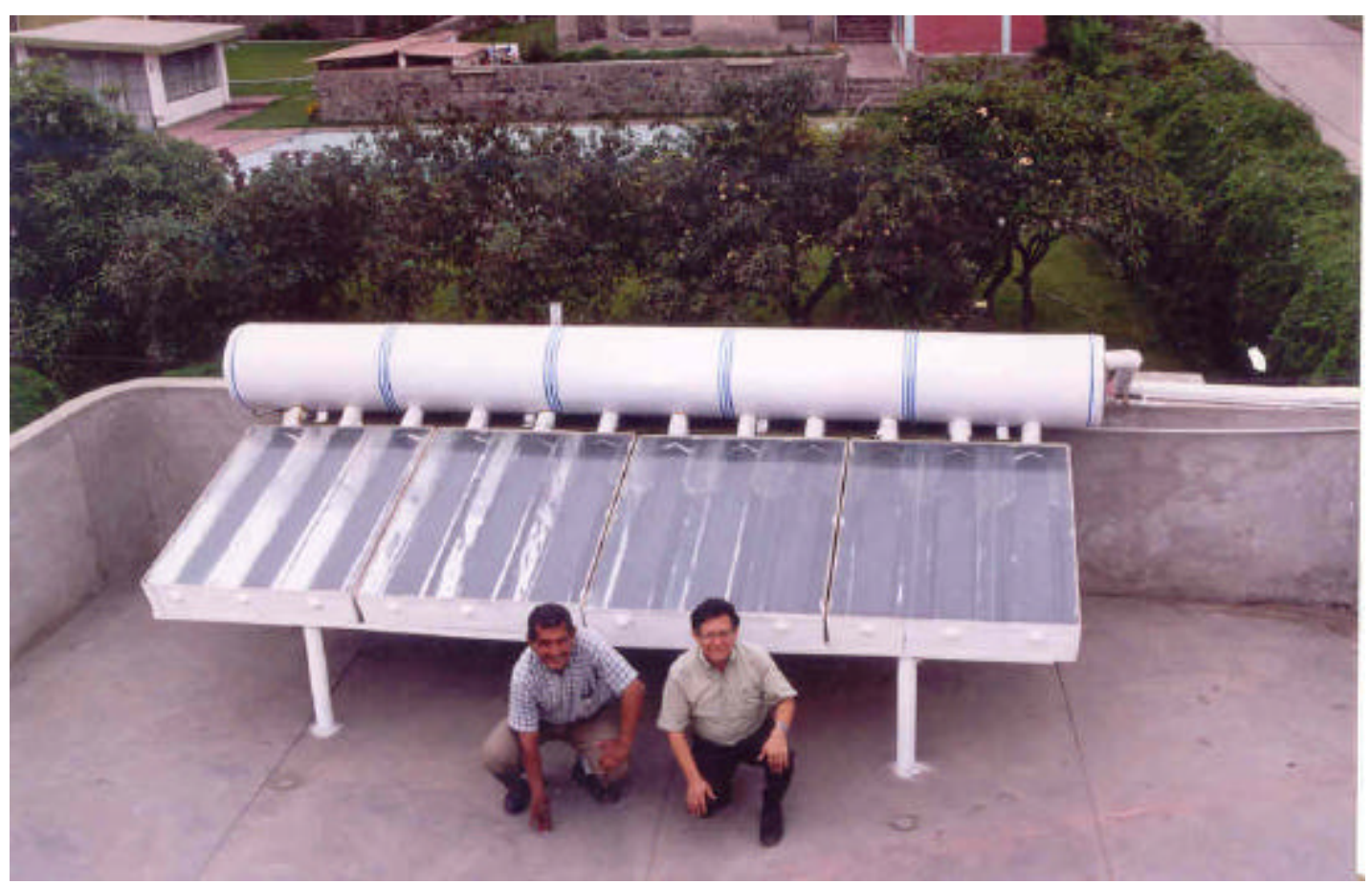

Figura 2. Vista panorámica de la therma solar en el Hotel de Yangas. Fuente: Elaboración propia, 2006. 
dibujó). Se hicieron 3 moldes CPC en MAPRESA para cada caja, cada molde tiene 3 CPC y se disponen de tal manera que uno está en la parte superior, otro en el medio y otro en la parte inferior, de tal manera que la lámina de aluminio pulido al asentarse firmemente en ellas, tome la forma parabólica compuesta. Las cajas tienen 1,25 m de largo por 1,00 m de ancho, en ese metro de ancho se dispusieron 3 CPC de 33 centímetros cada uno. En la cara superior e inferior de cada caja se pegó internamente papel aluminio para que refleje hacia adentro toda luz que en ellas incide.

La therma eléctrica se mandó construir con 12 tubos de $3 / 4$ de pulgada en su parte inferior (en la figura 1 sólo se dibujaron 3 ) estos tubos fueron conectados mediante pequeñas mangueras que resisten alta temperatura a los 12 tubos de cobre que salen de las 4 cajas ( 3 por caja), se aseguraron con abrazaderas y se sellaron con un pegamento rojo especial para manguera de alta temperatura.

El agua al ser calentada en los tubos de cobre por el sol sube por convección y se aloja en la parte superior de la therma, mientras que el agua fría que se encuentra en la parte inferior de la therma cae por gravedad por ser más densa hacia la parte inferior de los tubos, se calienta y vuelve a subir, así se produce un reciclaje que calienta toda el agua de la therma.

En la cara lateral derecha de la therma (de forma circular) salen 2 tubos: uno en la parte superior, por donde sale el agua caliente que se ha alojado en lo más alto del cilindro, rumbo a la ducha donde se recibe el agua caliente para bañarse; otro tubo en la parte inferior sirve para introducir el agua fría en la therma cada vez que sale agua caliente (para completar el volumen de agua de la therma), este tubo tiene una válvula check que permite ingresar el agua a la therma pero que impide que el agua caliente de la therma salga hacia la tubería de agua fría.

En la instalación: el tubo de agua caliente así como las mangueras y tubos de cobre externo por donde circula agua caliente, se protegen con tubos envolventes de poliuretano o lana de vidrio. Las 4 cajas colectoras se colocan con un ángulo de inclinación que depende de la latitud del lugar, en este caso para Lima corresponde 12 grados, la inclinación se apunta hacia el polo norte con ayuda de una brújula. Esta es la posición que permite que los colectores que van a estar fijos en esa posición todo el tiempo, reciban en promedio la mayor cantidad posible de energía del sol a lo largo del año.
CONCLUSIONES

La therma CPC de 500 litros, instalada en el Hotel Yangas es la materialización de la teoría CPC, allí hay agua caliente aún los días nublados, por lo que nunca fue necesario conectar la resistencia eléctrica en los tres años de funcionamiento.

Esta therma es mucho más barata que las thermas italianas o japonesas que se ofrecen en Lima a un alto costo y es tan eficiente como aquellas, mas que nada porque ellas vienen con la inclinación propia para Europa y por la forma como son construidas no se pueden adaptar a cada lugar. La nuestra sí, las mangueritas lo permiten.

Esta therma dio trabajo a muchos peruanos desde los que hicieron la therma eléctrica los que hicieron las 4 cajas de fibra de vidrio, los que soldaron los 12 tubos de cobre a las aletas, los que armaron y ensamblaron el sistema, hasta los que la transportaron y la subieron a la azotea del tercer piso (fue muy difícil subir los 4,60 metros de largo y 200 kilos de peso), terminando en los que la instalaron correctamente, demostrando que el peruano es hábil y es capaz de hacer obras de gran envergadura.

REFERENCIAS

BIBLIOGRÁFICAS

1. Jones, R.E. (1977). Optical properties of cylindrical elliptic concentrator, Proceedings American section of ISES, p, 36. 21.

2. Lunde, Peter J. (1980). Solar Thermal Engineering. John Wiley Sons. New York, USA. Primera edición.

3. Mac Daniels, D.K. et al. (1975). Enhanced Solar Energy Collection Using Reflector Solar thermal Collector Combinations.Solar Energy 17, 277-283.

4. Pérez Yebeña, Eduardo y Cruz Costa Jorge. (2006) ConversâoTérmica da Energia Solar. Edit SPESDER/INERI. Primera edición.

5. Rabl, Arí (1976). Comparison of Solar Concentrators. Solar Energy 18,93-111.

6. Shapiro, M.M. (1977). Non-focussing solar concentrators of casy manufacture. Solar Energy 19, 212-213. 